\title{
Recognition and Judgment of Electromagnetic Disturbance of Telemetry Equipment Based on Machine Learning
}

\author{
Luolan Yang ${ }^{1,}$, , Jiaqi Sun ${ }^{1}$, Linqiao $\mathrm{Jia}^{1}$ \\ ${ }^{1}$ Beijing Research Institute of Telemetry, Beijing 100094, China \\ aemail: 514058628@qq.com
}

Keywords: Support Vector Machine, Telemetry equipment, State identification.

\begin{abstract}
The complex electromagnetic environment will interfere with the performance of telemetry equipment, and the interference can not be directly observed and identified. The state of the data mining equipment is subject to the problem of electromagnetic interference, the use of support vector machine method for the equipment subject to interference and not subject to interference in a variety of state information feature extraction, training machine learning model, using the training model on the current Equipment status to test, to determine its interference situation. Based on the above methods, this paper designs and implements a machine learning-based interference monitoring and analysis program. The method is tested with the state data of the servo and baseband memory. The experimental results show that the method can detect the disturbance of the equipment and can be used to monitor the health of the system in real time.
\end{abstract}

\section{Introduction}

As the current development of aerospace industry is getting faster and faster, space telemetry system as an integral part of the space system, has become increasingly important. At present, the mission of spaceflight monitoring and control to multi-equipment, multi-type, multi-band development, monitoring and control system security, real-time, reliability and reusability put forward higher and higher requirements [1]. The ground observation satellites to data relay satellites, low earth orbit satellites to deep spacecraft, and various types of spacecraft launch, flighting and return process, they need tracking control the measurement and control system to control. Thus ensuring the spacecraft in accordance with the scheduled state and plan to complete the space mission [2]. Each measurement and control system need to track the implementation of spacecraft measurements, command transmission, voice transmission [3]

At present telemetry equipment already has automated test and fault diagnosis of these two modules, you can pre-task on the system performance self-test, but only after setting the specific parameters found in the system exception. These two modules do not take into account the impact of environmental factors on the system, and can not monitor the performance of the system in real-time during the task execution, or find the performance degradation of the system.

In this paper, the electromagnetic interference experiment is carried out through the real telemetry equipment. According to the AGC voltage, pitch angle and lockout lock state data collected by the servo and baseband, we can clean, classify, mark and data mining, and analyze the trend of the data . For abnormal changes in parameters to identify, so as to achieve the purpose of identifying abnormal state system.

In this paper, the machine learning and time series methods are used to analyze the experimental data in horizontal and vertical direction respectively. A software is designed to support the real-time detection and judge whether the current system is in normal state, whether it is affected by electromagnetic interference and whether the performance is degraded, so as to achieve the real-time monitoring of the health status of the system. Its significance lies in the timely detection of system anomalies, improve the reliability of the system measurement data.

\section{Design of the Interference fault identification based on SVM}

SVM uses non-linear mapping as the basic theory, and uses the inner product kernel function 
instead of the nonlinear mapping to the higher-order space. By finding the optimal hyperplane, the feature space is decomposed and the minimization of the confidence space is achieved by maximizing the distance from the hyperplane to the classification point. The training result is the support vector and the support vector is used for classifying and decision making. SVM is a small sample learning method with good effect, and has been widely used in signal processing, feature recognition and other fields.[4]

In this paper, we will judge whether the telemetry equipment is disturbed and predict it into a binary classification problem. The training set is constructed by collecting the equipment state data and annotating the interference situation. The data of the training set are trained to obtain the optimal classification plane, and then the state data of the telemetry system is used as the test set, and the trained SVM model is used to classify it.

In order to obtain better classification results, this paper improves the accuracy of using machine learning methods to identify the interference in the measurement and control system through data mining and cleaning, feature correlation analysis and machine learning model parameter adjustment.

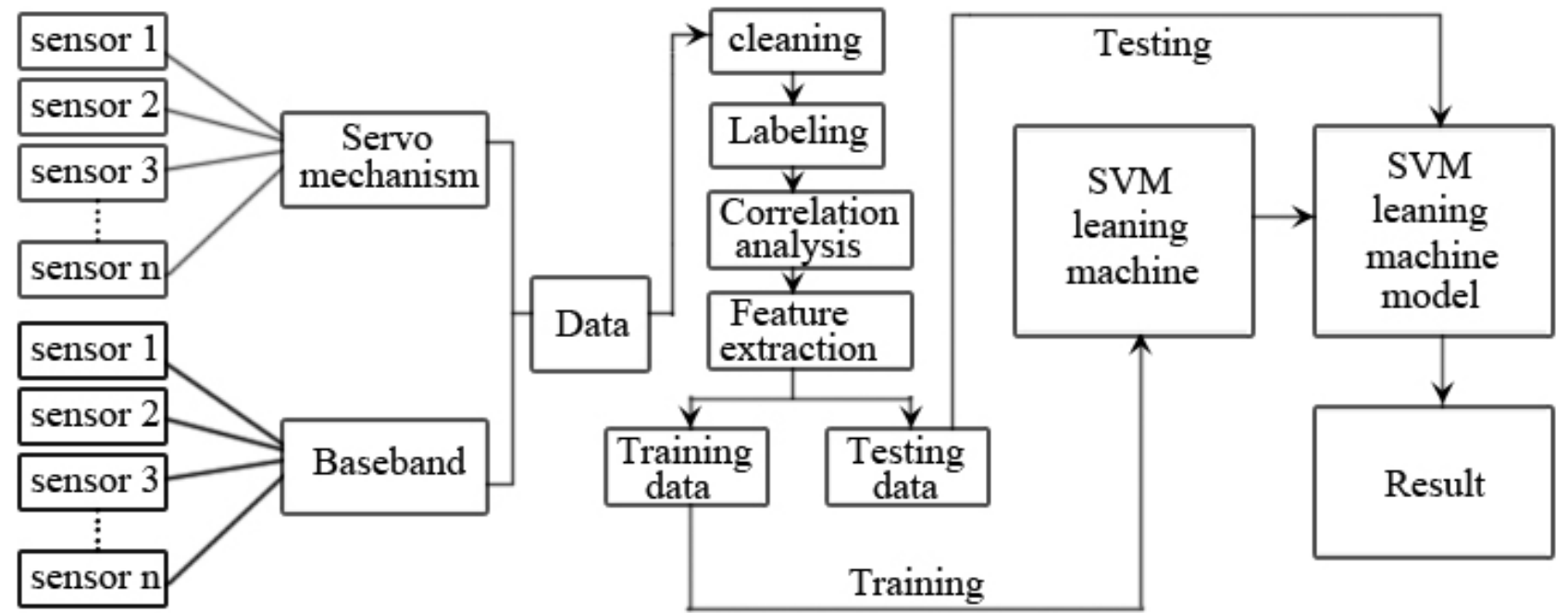

Fig.1. The Process of the Interference fault identification based on SVM

\section{Labeling and cleaning of interference data}

The mining of data features is the most basic and time-consuming work when machine learning is used to predict data. Since SVM is a machine learning method that needs to be supervised, its training set needs to be labeled data, so it is necessary to mark the state data collected by the device. And the data has some deviation from the surrounding points and a large number of points in the boot and shutdown there is no sense of training data for the training set will affect the training results of the meaningless data need to clean and remove the data and, The data is adjusted to the data format that machine learning can use.

Table 1 Part of the data measured by the servo under normal operation of the equipment

\begin{tabular}{c|c|c|c|c|c|c|c|c}
\hline \hline Time & $\begin{array}{c}\text { Real -time } \\
\text { angle A }\end{array}$ & $\begin{array}{c}\text { Command } \\
\text { angle A }\end{array}$ & $\begin{array}{c}\text { Angular } \\
\text { error }\end{array}$ & $\begin{array}{c}\text { Antenna } \\
\text { speed }\end{array}$ & lock & $\begin{array}{c}\text { AGC } \\
\text { Voltage }\end{array}$ & $\begin{array}{c}\text { Error } \\
\text { voltage A }\end{array}$ & $\begin{array}{c}\text { Error } \\
\text { voltageE }\end{array}$ \\
\hline 168-15:06:30.120 & 96.432 & 96.432 & 0 & 0 & 1 & 2.08 & 1.58 & -0.36 \\
\hline 168-15:06:30.130 & 96.443 & 96.443 & 0 & 0 & 1 & 2.11 & 1.74 & -0.37 \\
\hline 168-15:06:30.140 & 96.454 & 96.454 & 0 & 0 & 1 & 2.08 & 1.9 & -0.35 \\
\hline 168-15:06:30.150 & 96.465 & 96.465 & 0 & 0 & 1 & 2.08 & 2.05 & -0.37 \\
\hline 168-15:06:30.160 & 96.476 & 96.476 & 0 & 0 & 1 & 2.08 & 2.2 & -0.38 \\
\hline 168-15:06:30.170 & 96.487 & 96.487 & 0 & 0 & 1 & 2.04 & 2.38 & -0.37 \\
\hline 168-15:06:30.180 & 96.498 & 96.498 & 0 & 0 & 1 & 2.08 & 2.58 & -0.38 \\
\hline
\end{tabular}

Data marking and cleaning is an indispensable part of machine learning. Before using machine learning for feature recognition, we first need to manually divide the data into data and target, and explicitly describe the data items of the current phenomenon, and according to the required 
judgment, Given the meaning of these data items target, target that we need to machine the results of discrimination, this process is the process of marking the data.

Table 2 Part of the data measured by the servo in the event of abnormal operation of the equipment

\begin{tabular}{c|c|c|c|c|c|c|c|c}
\hline \hline Time & $\begin{array}{c}\text { Real -time } \\
\text { angle A }\end{array}$ & $\begin{array}{c}\text { Command } \\
\text { angle A }\end{array}$ & $\begin{array}{c}\text { Angular } \\
\text { error }\end{array}$ & $\begin{array}{c}\text { Antenna } \\
\text { speed }\end{array}$ & lock & $\begin{array}{c}\text { AGC } \\
\text { Voltage }\end{array}$ & $\begin{array}{c}\text { Error } \\
\text { voltage A }\end{array}$ & Error voltage E \\
\hline 168-15:03:53.000 & 100.1 & 100.102 & 0.005 & 0 & 1 & 3 & 3.72 & 0.51 \\
\hline 168-15:03:53.010 & 100.11 & 100.113 & 0.005 & 0 & 1 & 2.46 & 3.69 & 0.5 \\
\hline 168-15:03:53.020 & 100.12 & 100.124 & 0.005 & 0 & 1 & 3.02 & 3.67 & 0.51 \\
\hline 168-15:03:53.030 & 100.13 & 100.135 & 0.005 & 0 & 1 & 2.36 & 3.66 & 0.5 \\
\hline \hline
\end{tabular}

\section{Feature Extraction Based on Correlation Analysis}

After marking and cleaning the data, it's necessary to select the characteristic data that can be used for data prediction. Before performing the correlation analysis, we should first determine which data volumes will be affected by electromagnetic interference, and those data will not be affected by electromagnetic interference. We make the angle of the antenna according to the same angular speed, turn a certain angle, and the equipment to make interference and are not subject to interference. Under normal conditions, the partial parameters change with time as shown in Fig 2:

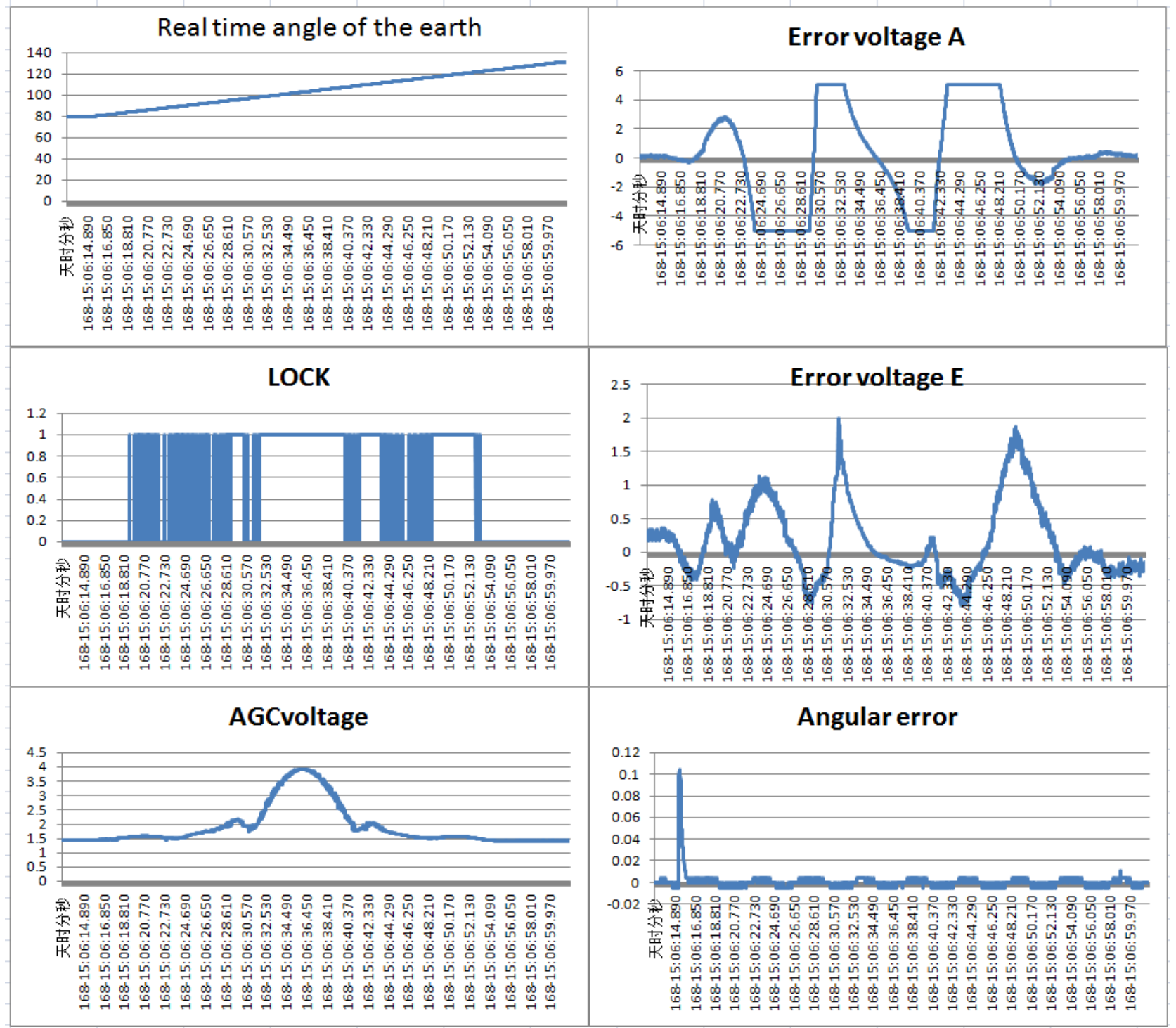

Fig.2. The change trend of each parameter under the normal operation condition of the equipment Abnormal state of some parameters with time trends shown in Figure 3. 


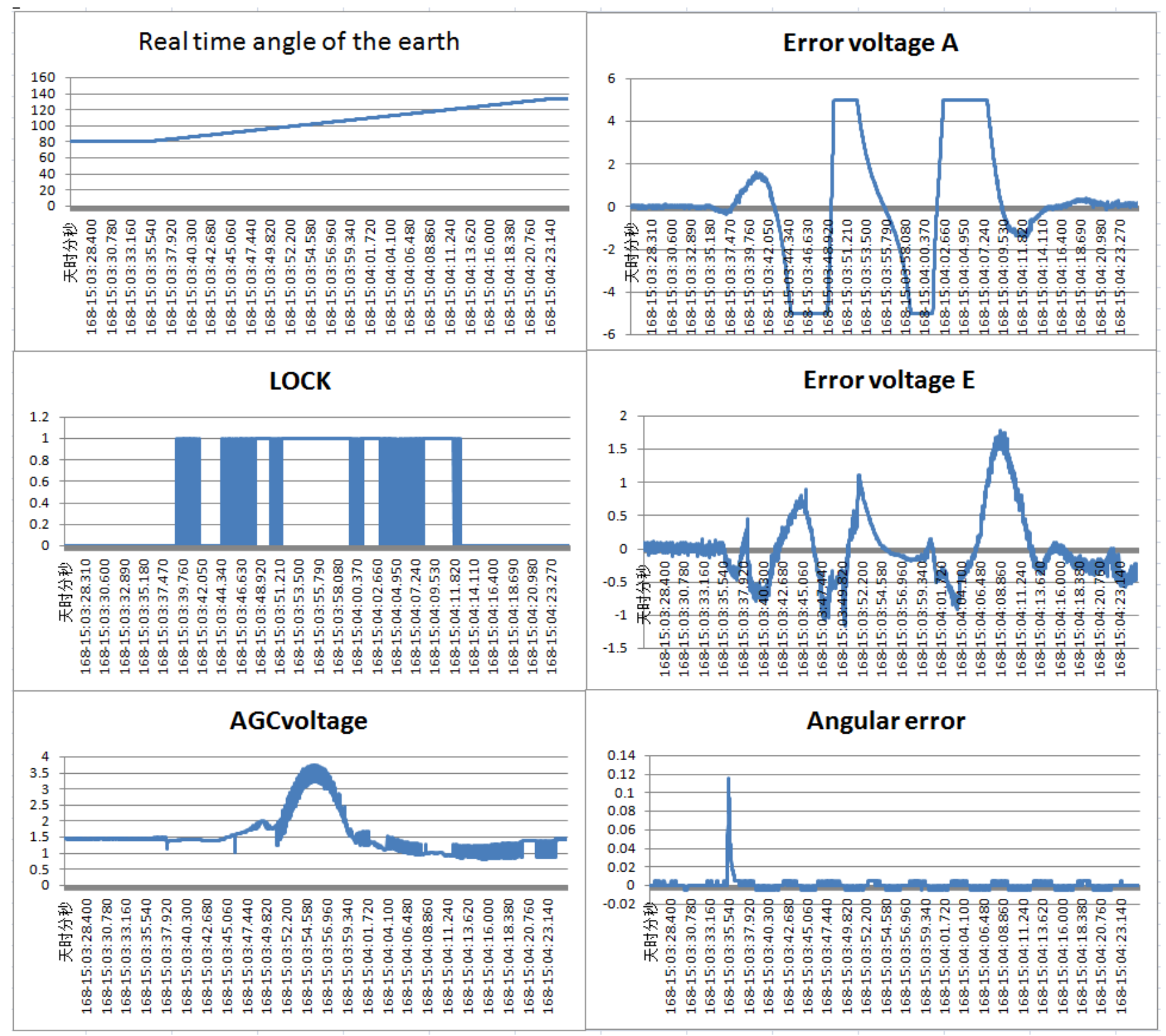

Fig.3. The parameters of the equipment under the interference situation with time trends

It can be seen from the curves of the parameters in the figure that the real-time angle relative to the earth is in fixed slope positively correlated with the time, because the antenna is rotating at uniform angular velocity.So we can use the real-time angle to represent the change of time. Using the real-time angle as the independent variable, we can easily compare the difference of the feature data under the same condition of the independent variable and the case of interference and interference .

In the selection of feature data, we should select the data related to the prediction target as the feature data, we need to first calculate the correlation between the feature data and the predicted target, and then compare the two kinds of feature data in the same real time angle of the earth , then find the differences.

Correlation analysis is to study the phenomenon of the existence of a dependency between the methods. In the case of equipment state classification and judgment using nuclear machine learning, it is necessary to first analyze the correlation and determine which measured data may be related to the data item which is finally desired to be judged.

The data collected include AGC voltage, the real time angle of the earth, baseband locking, angular error, error voltage E, error voltage A and other characteristics of the data. After analyzing the correlation between the real time angle of the earth and other parameters, the AGC voltage, real time angle of the earth, the baseband locking condition and the error voltage A are selected as the basis of the electromagnetic interference. 


\section{Optimization Algorithm Based on Feature Sequence Recognition}

Since the method of machine learning is affected by the randomness of the feature point distribution, the result of the discrimination of a single point is erroneous. However, this random error can be reduced if a set of feature points of a certain class is judged. According to the analysis of the data, as we can see in figure 4 that the appearance of interference is continuous, and in the vicinity of the point of interference, many disturbed points should appear continuously. And a plurality of successive points may be represented by a sequence of features. Therefore, we can identify the interference state of the device by means of the feature sequence identification method.

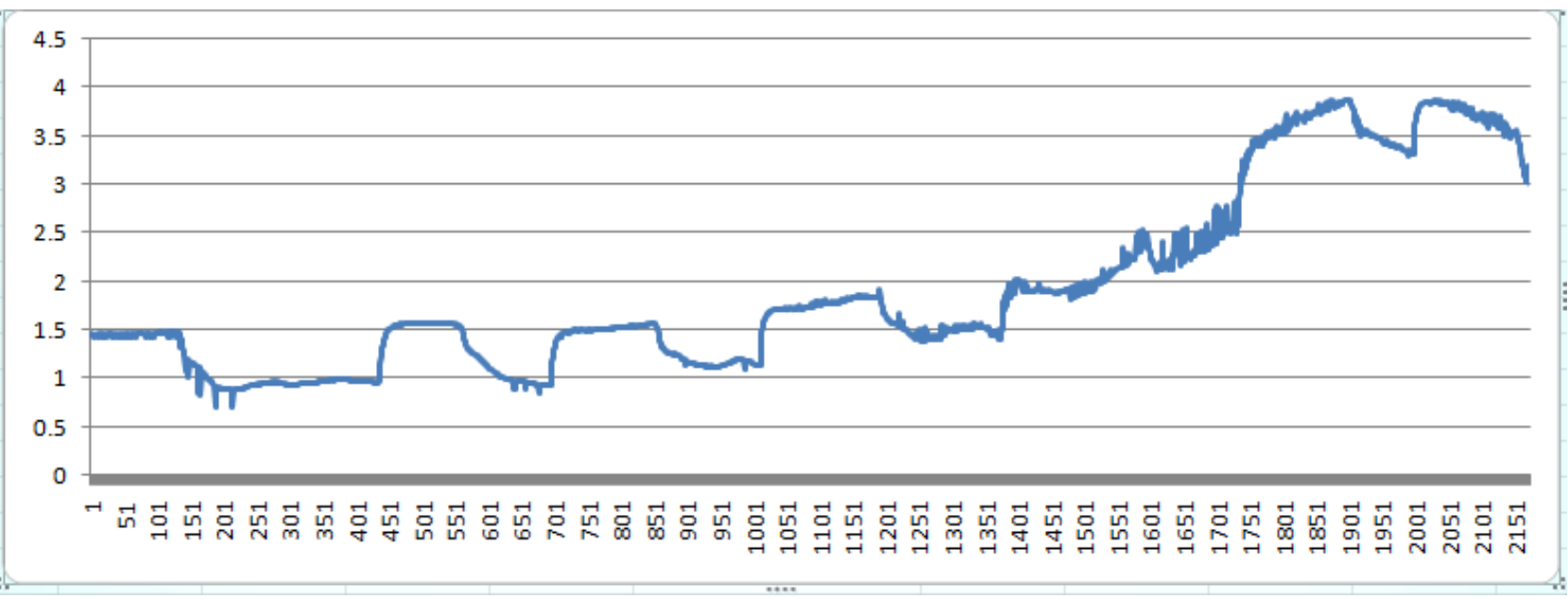

Fig.4. The part of the data measured by the servo when the equipment is intermittently disturbed

The sequence S contains two kinds of point,one kind is device that is subject to interference and the other kind is the device that isn't subject to interference. As the process that the device that is subject to interference should be a continuous process, if the device is subject to interference,the point in $\mathrm{S}$ should be :

(1) Have characteristics of disturbed points.

At this time, the model of machine learning will classify most of the points as disturbed by averaging the discriminant results of multiple points and calculating the Euclidean distance from the mean to the two target results. If the distance is small, the discriminant result Closer.

(2) Sequence S should have some statistical characteristics.

According to the previous data analysis, when the telemetry equipment is disturbed, the AGC voltage measured by the device will appear a slight jump, and its mean value will be decreased.

Here we use the average jump rate to identify the interference of the feature sequence. The process is as follows:

(1) Take the first two points in the feature sequence, calculate the absolute value of the difference and record

(2) Take the third point and compute the absolute value of the difference from the second and record

(3) Take the last point in the feature sequence, sum all the differences and take the average

According to the actual calculation, it is found that the average jump rate in the normal state is 0.01 , and the average jump rate in the abnormal state is 0.07 . Therefore, for any feature sequence, the average jump rate can be calculated, and as a basis to determine its interference state.

\section{Experimental verification}

The test environment is as follows: The position of the beacon is azimuth $A=103$ degrees, pitch angle $\mathrm{E}=90$ degrees, distance $84 \mathrm{~m}$ (the distance between the two sides of the beacon), the beacon transmitting signal strength is $33 \mathrm{dBm}$. The device uses the manual alignment mode, the azimuth slowly from 80 degrees to 130 degrees, analog equipment at various angles tracking status. As the observation angle bias is large, the measured signal strength will also change according to the angle 
changes. Therefore, the beacon signal strength is set to a fixed value. Thereby observing the device AGC voltage, angular error, pitch angle, locking out of lock situation.

\section{Experimental data acquisition and labeling}

(1) In the equipment normal state test, the equipment adopts the manual alignment mode, and adjusts the azimuth angle slowly from 80 degrees to 130 degrees. The servo stores the normal operation and operation data of the equipment at the speed of 10 times per second. The status of the device is changing, which is provided by the servo and baseband systems, as a positive example in the training set.

(2) In the electromagnetic interference environment, the manual alignment mode, the azimuth slowly from 80 degrees to 130 degrees, the whole process of setting the interference source on the telemetry equipment, electromagnetic interference, the same servo and baseband When the device is disturbed, the data is stored as the original data in the interference state of the device. The data is used as a counter example in the training set.

(3) In the environment of intermittent electromagnetic interference, the device adopts manual alignment mode, modulates the azimuth angle slowly from 80 degrees to 130 degrees, intermittently sets the interference source to interfere with the equipment, and finally records the mixed state data of the equipment. The data as a validation set of raw data, the part of the data shown in Table 3 , observe the data in the table, the individual data AGC voltage remains stable, and individual data is intermittent AGC voltage jump phenomenon. These phenomena are similar to the data representation in the abnormal state of the device. These data are used as test sets.

Table 3 Part of the data measured by the servo when the equipment is intermittently disturbed

\begin{tabular}{c|c|c|c|c|c|c|c|c}
\hline \hline Time & $\begin{array}{c}\text { Real -time } \\
\text { angle A }\end{array}$ & $\begin{array}{c}\text { Command } \\
\text { angle A }\end{array}$ & $\begin{array}{c}\text { Angular } \\
\text { error }\end{array}$ & $\begin{array}{c}\text { Antenna } \\
\text { speed }\end{array}$ & lock & $\begin{array}{c}\text { AGC } \\
\text { Voltage }\end{array}$ & $\begin{array}{c}\text { Error } \\
\text { voltag } \\
\text { e A }\end{array}$ & $\begin{array}{c}\text { Error } \\
\text { voltage E }\end{array}$ \\
\hline $168-15: 00: 02.300$ & 82.892 & 82.897 & 0.005 & 0 & 0 & 1.48 & -0.21 & -0.3 \\
\hline $168-15: 00: 02.310$ & 82.903 & 82.908 & 0.005 & 0 & 0 & 1.48 & -0.2 & -0.31 \\
\hline $168-15: 00: 02.320$ & 82.914 & 82.919 & 0.005 & 0 & 0 & 1.42 & -0.23 & -0.3 \\
\hline $168-15: 00: 02.330$ & 82.925 & 82.93 & 0.005 & 0 & 0 & 1.42 & -0.23 & -0.3 \\
\hline $168-15: 00: 02.340$ & 82.936 & 82.941 & 0.005 & 0 & 0 & 1.48 & -0.25 & -0.3 \\
\hline $168-15: 00: 02.350$ & 82.947 & 82.952 & 0.005 & 0 & 0 & 1.47 & -0.23 & -0.32 \\
\hline $168-15: 00: 02.360$ & 82.963 & 82.963 & 0 & 0 & 0 & 1.45 & -0.23 & -0.34 \\
\hline $168-15: 00: 02.370$ & 82.974 & 82.974 & 0 & 0 & 0 & 1.33 & -0.28 & -0.31 \\
\hline $168-15: 00: 02.380$ & 82.985 & 82.985 & 0 & 0 & 0 & 1.41 & -0.21 & -0.3 \\
\hline $168-15: 00: 02.390$ & 82.996 & 82.996 & 0 & 0 & 0 & 1.36 & -0.19 & -0.33 \\
\hline $168-15: 00: 02.400$ & 83.002 & 83.007 & 0.005 & 0 & 0 & 1.32 & -0.21 & -0.33 \\
\hline $168-15: 00: 02.410$ & 83.013 & 83.018 & 0.005 & 0 & 0 & 1.28 & -0.18 & -0.28 \\
\hline $168-15: 00: 02.420$ & 83.024 & 83.029 & 0.005 & 0 & 0 & 1.26 & -0.2 & -0.29 \\
\hline \hline
\end{tabular}




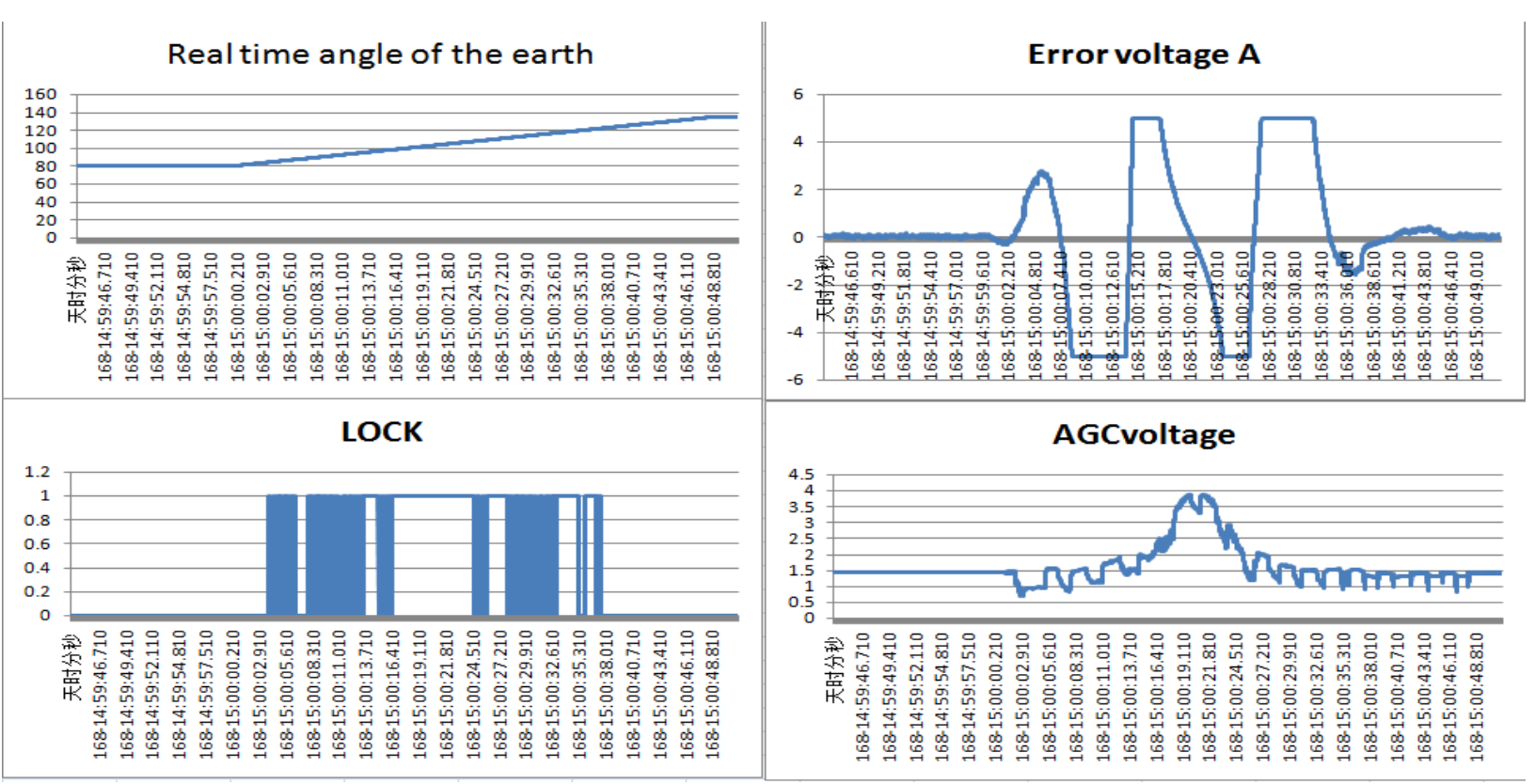

Fig.5. The part of the data measured by the servo when the equipment is intermittently disturbed

\section{Design and Implementation of Experimental Program}

Program get data from the log data monitoring system. The function of the program is to determine whether the device is subject to electromagnetic interference using the SVM machine learning model. The program includes a log data acquisition and processing module, a support vector machine training module, a system state judgment module and a result display module.

Log data acquisition and processing module's main function is to read the file from the monitoring system. The module extracts the data in the file as single data item, and transforms text format data items into Floating-point number form, then create a state matrix to preserve all the state information.

The main function of the support vector machine's training module is to utilize the state information of the equipment saved in the state matrix . According to the support vector machine's training method, the support vector machine can be trained to get the interference state classification model.

The state module obtains the current state of the equipment from the monitoring system, and tests the current state with the trained SVM. The test result is returned by the support vector machine, and then the feature sequence is analyzed according to the test result. The interference state is given.

The function of the display module is to display the classification results.

The program is programmed in Python, which can read the state data, support the training and forecasting of SVM, evaluate the prediction result and optimize the feature sequence by using the method of feature sequence.

\section{Training of Machine learning model}

In this experiment, the use of nuclear machine learning state of the device classification and determination, we must first clear the following: 1, the availability of the current state of the equipment characterization data; 2, the results of the items to be determined; State data and the determination of the relationship between items. The state data is the main data source of training set and validation set. The training set and validation set need to be classified. First, we will test the equipment under the interference of data and equipment to work properly separate data, respectively, marked 0 and 1.0 on behalf of the equipment is normal, 1 on behalf of abnormal state equipment. Secondly, we use the training set to training SVM as the core training program to get a classification model of the vector machine, and finally we use the validation set of data to verify the test, the 
program gives the test results and the original mark to do comparison, Accuracy, recall rate and f1-score, to measure the classification effect.

\section{Classification of machine learning models}

For binomial kernel machine learning, performance can be evaluated using accuracy, recall, and kernel matrices. The whole training and testing process takes 12.799990s, the result of the confusion matrix is:

Table 4 Confusion Matrix

\begin{tabular}{l|c|c}
\hline \hline & Actual undisturbed & Actual interference \\
\hline The forecast is undisturbed & 4348 & 299 \\
\hline Predictions are subject to interference & 543 & 1557 \\
\hline \hline
\end{tabular}

Table 5 Results report

\begin{tabular}{l|c|c|c|c}
\hline \hline & Accuracy & Recall rate & f1-score & Actual number \\
\hline Undisturbed & 0.94 & 0.89 & 0.91 & 4891 \\
\hline Subject to interference & 0.74 & 0.84 & 0.79 & 1856 \\
\hline \hline
\end{tabular}

It can be seen from the results, the recognition accuracy and the recall rate of the undisturbed state are high, and that of the subject to interference state is relatively low. The average accuracy is more than $80 \%$,it indicates that the program can automatically identify and analyze the current state of the device is abnormal, and timely to give abnormal alarm prompts.

\section{Summary and Prospect}

At present, the telemetry system can monitor the attitude of the spacecraft more comprehensively, but its condition monitoring and analysis should be improved. This paper designs and realizes the automatic diagnosis and diagnosis program of the telemetry system based on machine learning, and carries on the equipment state diagnosis experiment under the abnormal condition of the electromagnetic interference. The experimental results show that this method can more accurately identify the current health condition of the telemetry system. Thus enhancing the reliability of the telemetry system itself, making the equipment in the implementation of telemetry tasks more reliable, the accuracy of the measured data has been better protected. It is hoped that the telemetry system not only records its own state data, but also can increase the real-time monitoring and analysis of the key indicators of each subsystem, from the current after-maintenance to the condition of repair.

\section{References}

[1] Gang Wang, XiaoYue Wu.The Composition and Development of Spaceflight TT\&C Systems in USA [J].National Defense Science \& Technology, 2010, 31(5):87-91

[2] Yuncai Liu. Telemetering and remote control system[M].National Defence Industry Press,2000.

[3] Xiankui Sun, Lan Qin. Development Status and Trend of Remote Measurement and Control Technology[J].Chinese Journal of Scientific Instrument. 2004, 25(z3):562-564.

[4]Gang Ding. Research on Environment Perception and Object Recognition for Mobile Robot Based on Support Vector Machine[D].Anhui: University of Science and Technology of China, 2009:10-12. 